\title{
Common genomic elements promote transcriptional and DNA replication roadblocks
}

\author{
Kevin Roy, ${ }^{1,2}$ Jason Gabunilas, ${ }^{1}$ Abigail Gillespie, ${ }^{1}$ Duy Ngo, ${ }^{1}$ and Guillaume \\ F. Chanfreau ${ }^{1,2}$ \\ ${ }^{1}$ Department of Chemistry and Biochemistry, University of California, Los Angeles, Los Angeles, California 90095-1569, USA; \\ ${ }^{2}$ Molecular Biology Institute, University of California, Los Angeles, Los Angeles, California 90095-1570, USA
}

\begin{abstract}
RNA polymerase II (Pol II) transcription termination by the Nrdlp-Nab3p-Senlp (NNS) pathway is critical for the production of stable noncoding RNAs and the control of pervasive transcription in Saccharomyces cerevisiae. To uncover determinants of NNS termination, we mapped the 3'-ends of NNS-terminated transcripts genome-wide. We found that nucleosomes and specific DNA-binding proteins, including the general regulatory factors (GRFs) Reblp, Raplp, and Abflp, and Pol III transcription factors enhance the efficiency of NNS termination by physically blocking Pol II progression. The same DNAbound factors that promote NNS termination were shown previously to define the 3'-ends of Okazaki fragments synthesized by Pol $\delta$ during DNA replication. Reduced binding of these factors results in defective NNS termination and Pol II readthrough. Furthermore, inactivating NNS enables Pol II elongation through these roadblocks, demonstrating that effective Pol II termination depends on a synergy between the NNS machinery and obstacles in chromatin. Consistent with this finding, loci exhibiting Pol II readthrough at GRF binding sites are depleted for upstream NNS signals. Overall, these results underscore how RNA termination signals influence the behavior of Pol II at chromatin obstacles, and establish that common genomic elements define boundaries for both DNA and RNA synthesis machineries.
\end{abstract}

[Supplemental material is available for this article.]

Partitioning the genome into functional genes depends on the recognition of transcription initiation and termination sites by RNA polymerase II (Pol II). Pol II transcription initiation is largely specified by the establishment of nucleosome-depleted regions (NDRs) (Neil et al. 2009; Venters and Pugh 2009; Xu et al. 2009). In contrast, Pol II transcription termination is directed by signals in the nascent RNA that recruit termination factors (Steinmetz et al. 2001; Proudfoot 2011). The subsequent interaction of termination factors with Pol II promotes conformational changes that trigger Pol II release (Richard and Manley 2009; Skourti-Stathaki et al. 2011; Zhang et al. 2015). Transcription termination plays important roles in preventing transcriptional interference, in recycling Pol II for subsequent rounds of transcription, and in promoting gene loop formation (Grzechnik et al. 2014). The coupling of transcriptional termination with 3 '-end processing also influences RNA stability and nuclear export (Porrua and Libri 2015).

In the yeast Saccharomyces cerevisiae, two primary termination pathways exist for Pol II: the canonical mRNA cleavage and polyadenylation (CPA) pathway and the Nrd1p-Nab3p-Sen1p (NNS) termination pathway, which functions in the termination of stable ncRNAs and cryptic transcripts (Wyers et al. 2005). Nrd1p and Nab3p recognize tetrameric motifs in the nascent RNA and recruit the Sen $1 p$ helicase, whose subsequent interaction with Pol II promotes termination (Steinmetz et al. 2001; Carroll et al. 2007). The NNS pathway has been shown to terminate transcription across broad genomic windows of ill-defined length, resulting in widely heterogeneous 3 '-ends (Wyers et al. 2005). Recent work demonstrated existence of a kinetic competition between Pol II elongation and Sen1p-mediated termination (Hazelbaker et al.

Corresponding author: guillom@chem.ucla.edu

Article published online before print. Article, supplemental material, and publication date are at http://www.genome.org/cgi/doi/10.1101/gr.204776.116.
2013). In addition, two other termination pathways have been described. The first involves cotranscriptional RNA cleavage by the yeast RNase III homolog Rnt1p followed by destabilization of Pol II by the Rat1p exonuclease (Ghazal et al. 2009; Rondón et al. 2009). The second involves the DNA-binding protein Reb1p, which triggers roadblock-dependent termination of RNA Pol II in a mechanism proposed to involve Rsp5p-mediated ubiquitylation of Pol II (Colin et al. 2014). The generality of this mechanism as well as why a substantial fraction of Reb1p binding sites do not appear to roadblock Pol II remains to be established.

The 3 '-ends of nascent RNAs released by the NNS and Rnt1pmediated termination pathways are targeted for trimming or degradation by the combined action of the Trf4p-Air2p-Mtr4p polyadenylation (TRAMP) complex and the nuclear exosome (LaCava et al. 2005; Vanácová et al. 2005; Wyers et al. 2005). Previous tiling array and RNA-seq studies utilized cells deficient for nuclear exosome function and characterized broad genomic regions of up-regulation to identify NNS-targeted transcripts. These studies lacked the resolution to identify precise 3 '-ends generated by NNS termination, and it is currently unknown how NNS directs the release of Pol II at specific termination sites. As NNS signals are tetrameric motifs present in virtually all Pol II transcripts, we reasoned there might be additional determinants controlling NNS specificity. To address these questions, we profiled the sites of NNS termination genome-wide by sequencing 3 '-ends of transcripts after nuclear exosome inactivation.

C 2016 Roy et al. This article is distributed exclusively by Cold Spring Harbor Laboratory Press for the first six months after the full-issue publication date (see http://genome.cshlp.org/site/misc/terms.xhtml). After six months, it is available under a Creative Commons License (Attribution-NonCommercial 4.0 International), as described at http://creativecommons.org/licenses/by-nc/ $4.0 \%$ 


\section{Results}

Genome-wide distribution of nuclear-exosome targeted poly (A) ${ }^{+}$RNA 3'-ends (xPATs)

In the absence of the Rrp6p exonuclease, RNA Pol II transcripts terminated by the NNS pathway accumulate as polyadenylated species (Wyers et al. 2005). To globally map NNS termination sites, we profiled 3 -ends of poly (A) ${ }^{+}$RNAs in wild-type (WT) cells and cells carrying a deletion of RRP6 (rrp64) using oligo-d(T)-based 3 '-end sequencing (see Methods) (Fig. 1A). In accordance with previous studies, $>90 \%$ of poly (A) ${ }^{+}$tags (PATs) in WT cells mapped to 3' UTRs (Fig. 1B; Ozsolak et al. 2009; Pelechano et al. 2013; Wilkening et al. 2013). RRP6 deletion increased PATs from known NNS targets, including cryptic unstable transcripts (CUTs), stable unannotated transcripts (SUTs), and Xrn1-sensitive unstable transcripts (XUTs) (Fig. 1B; Neil et al. 2009; Schneider et al. 2012; Webb et al. 2014). Overall, 8956 PATs up-regulated greater than or equal

A

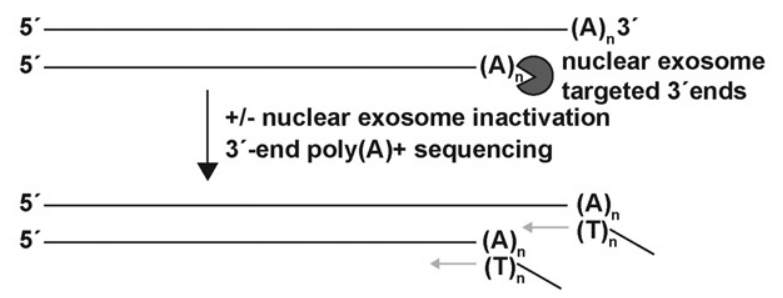

B
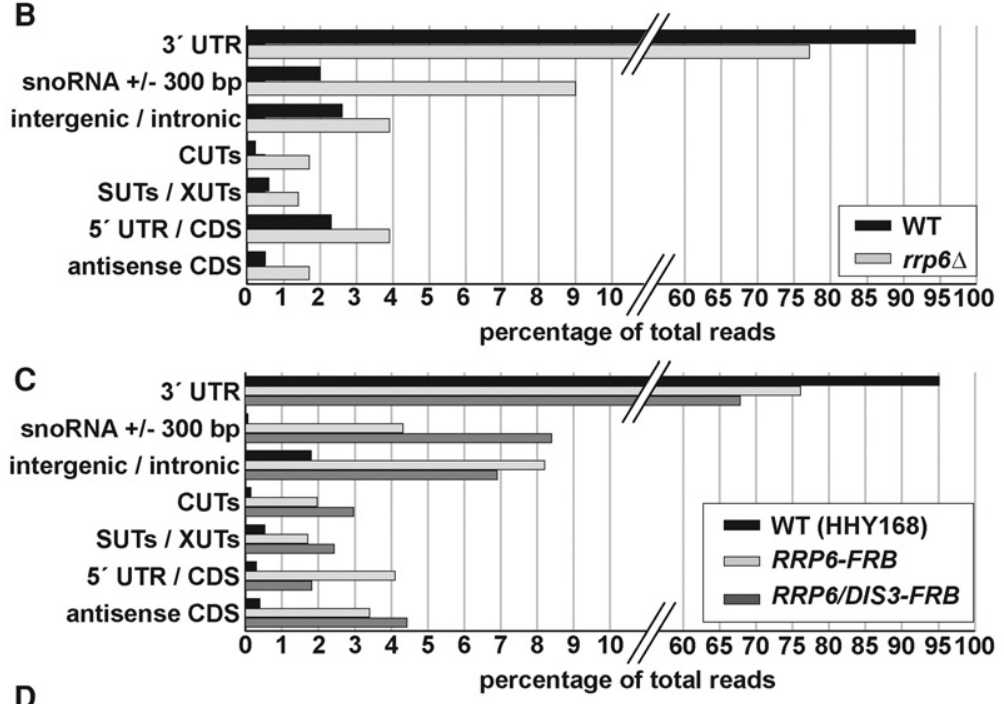

D

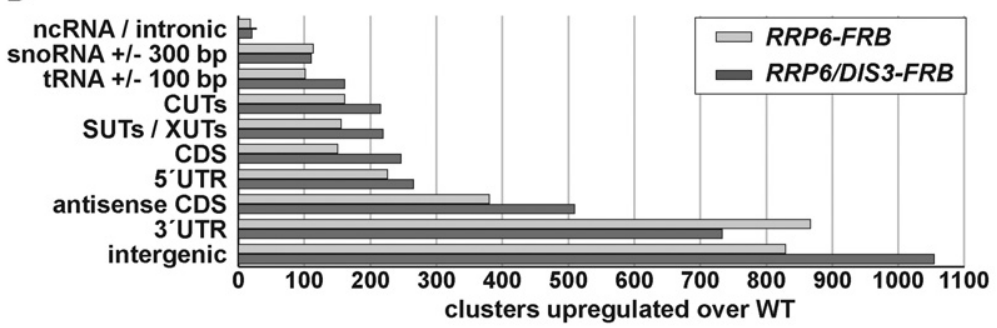

Figure 1. Distribution of poly $(A)^{+}$RNA $3^{\prime}$-end tags (PATs) upon inactivation of the nuclear exosome. (A) Strategy for mapping $3^{\prime}$-ends of RNAs targeted by the nuclear exosome. (B) Global distribution of PATs in WT and rrp6 4 . PATs mapping to the indicated regions were tallied and grouped by the indicated genomic annotations. (C) Global distribution of PATs upon nuclear depletion of Rrp6p or codepletion of Rrp6p and Dis3p by the anchor away system. $(D)$ Distribution of PATs clusters up-regulated in RRP6-FRB and RRP6/DIS3-FRB strains relative to WT (see Methods). to fivefold in $\operatorname{rrp6\Delta }$ over WT clustered into 1207 distinct regions (minimum of two reads per million [rpm]; see Methods) (Supplemental Table S1). A substantial fraction of these clusters from unannotated intergenic regions, indicating cryptic due to more sensitive detection of shorter transcripts by $3^{\prime}$-end se(the standard RNA-seq. We also identified numerous role in degrading and processing these distinct classes of trancripts, respectively.

The majority of snoRNA transcription units undergo NNS termination followed by exosome trimming (Carroll et al. 2004). In p $6 \Delta$, snoRNAs accumulate poly $(\mathrm{A})^{+} 3^{\prime}$-extensions corresponding products (Allmang et al. 1999; van Hoof et al. 2000; Grzechnik Kufel 2008). Meta-analysis of all snoRNA downstream regions revealed a broad window of PATs with the characteristic heterogeneous lengths generated by NNS termination (Supplemental Fig. S1), consistent with previous observations (Hazelbaker et al. 2013). To independently validate these results with a different platform, we performed direct RNA sequencing on the same RNA samples (Supplemental Fig. S1A). A lower percentage of total reads mapping to $3^{\prime}$ UTRs was detected with direct RNA sequencing possibly due to its shorter median read length ( $>50 \%$ of reads $<25 \mathrm{bp}$ ) and higher error rate (Ozsolak 2014). Nonetheless, the two methods demonstrated excellent agreement regarding the relative positions of PATs downstream snoRNAs (Supplemental Fig. S1B).

We noticed two prominent peaks downstream from snoRNAs: one at the mature 3 '-ends and another $25 \mathrm{nt}$ downstream (Supplemental Fig. S1B). This distance matches the length of singlestranded RNA threaded through the central channel of the exosome to the active site of the Dis3p nuclease (Makino et al. 2013). During the processing of the 5.8S rRNA precursor, Dis3-mediated nuclear exosome trimming stalls $\sim 30 \mathrm{nt}$ from the mature 3 '-end, and Rrp6p is required for efficient trimming of the remaining nucleotides (Briggs et al. 1998). To test whether the $+25 \mathrm{nt}$ peak represents a Dis3p-dependent processing intermediate, we employed the anchor away system to deplete either Rrp6p or both Rrp6p and Dis3p from the nucleus (Mitchell et al. 1997; Haruki et al. 2008). One hour of Rrp6p depletion resulted in a substantial increase in PATs from non-3' UTRs, while codepletion of Rrp6p and Dis3p resulted in further increase (Fig. 1C). Rrp6p depletion resulted in 11,881 up-regulated PATs clustering into 3247 regions, while Rrp6p/Dis3p depletion resulted in 17,690 up- 
regulated PATs (nuclear-exosome targeted PATs, or xPATs) clustering into 3316 regions (Fig. 1D; Supplemental Table S1). Importantly, Rrp6p nuclear depletion resulted in the same +25 nt snoRNA peak detected in $\operatorname{rrp6\Delta }$ (Supplemental Fig. S1C). This peak was markedly reduced by codepletion of Dis3p, confirming that it represents a Dis3p-dependent processing intermediate. For the remainder of this study, we analyzed xPATs accumulating from combined Rrp6p/Dis3p nuclear depletion to minimize levels of Dis3p-dependent processing intermediates and to enrich for primary products of NNS termination.

\section{xPATs provide a high-resolution view of NNS termination sites}

Next we tested the prediction that xPATs mark the actual sites of NNS termination. We analyzed snoRNA xPATs in greater detail, as snoRNA transcription units exhibit the most well-characterized NNS terminators (Carroll et al. 2004; Tudek et al. 2014). To inactivate NNS termination, we depleted the Sen1p helicase from the nucleus in addition to Rrp6p and mapped xPATs in a 1-kb window downstream (Fig. 2A). Ignoring the mature 3 '-end and +25 -nt processing peaks, nuclear depletion of either Rrp6p or both Rrp6p and Dis3p led to a similar xPATs pattern (Fig. 2A, i and ii; Supplemental Fig. S2). Codepletion of Sen1p and Rrp6p led to a loss of the xPATs centered around $+300 \mathrm{nt}$ and to an increase in signal further downstream, confirming that xPATs downstream from snoRNAs are generated by NNS. Importantly, the mature 3 '-end and +25 nt processing peaks were not reduced by Sen $1 \mathrm{p}$ depletion but rather slightly increased (Fig. 2A, i).

A recent report implicated a role for Rrp6p in promoting termination of a subset of NNS terminators (Fox et al. 2015). The mechanistic basis for this phenomenon was not elucidated, and it was not clear how the deletion of RRP6 affected termination globally. To rule out potential indirect effects of nuclear exosome inactivation on termination, we incorporated data from previous studies that mapped regions of NNS termination, by sequencing either RNA bound to Pol II (PAR-CLIP of the Pol II subunit Rpb2p), or DNA bound to Pol II (ChIP-seq of the Pol II subunit Rpb3p), respectively (Schulz et al. 2013; Schaughency et al. 2014). These studies identified locations where Pol II signal increased upon nuclear depletion of Nrd1p to infer regions in which NNS termination normally occurs. By meta-analysis, we confirmed that Nrd1p depletion resulted in an increase in Pol II PAR-CLIP and ChIP-seq in the snoRNA termination regions, with the PAR-CLIP signal generally dropping more rapidly in the 3 ' region than the ChIP-seq signal (Fig. 2A, ii and iii). Importantly, the majority of xPAT peaks coincided with the major increase in Pol II occupancy upon Nrd1p depletion, with closer agreement to the ChIP-seq data (Fig. 2A, ii). Analysis of individual examples showed that the xPATs reside in between the termination sites inferred in these previous studies (Supplemental Fig. S2). Notably, $>70 \%$ of the termination sites reported by Pol II PAR-CLIP were $>100$ bp upstream of those obtained by Pol II ChIP-seq (Schaughency et al. 2014), and 13 of the 45 termination sites determined by PAR-CLIP were actually assigned inside the snoRNA genes (Supplemental Table S2). Based on the comparison to these previous data sets, we conclude that XPATs provide an accurate location of the sites of NNS termination in WT cells.

Prior to 3 '-end trimming, NNS termination products acquire short oligo(A) tails in the range of one to 12 adenosines; in the absence of nuclear exosome activity, these tails are lengthened to $>20$ nt by sequential rounds of TRAMP activity (Wyers et al. 2005; Egecioglu et al. 2006). To confirm that xPAT oligo(A) tails are in- deed long enough to prime the oligo- $\mathrm{d}(\mathrm{T})_{18}$ employed in our protocol, we subjected total RNA from WT and RRP6/DIS3-FRB to in vitro polyadenylation, which universally added more than 100 adenosines (see Supplemental Methods). As expected, in vitro polyadenylation resulted in the mature 3 '-ends of snoRNAs dominating the signal (Supplemental Fig. S3, ii). Masking this signal revealed remarkable consistency between xPATs detected with and without in vitro polyadenylation (Supplemental Figs. S3, i and iii, S2). This confirmed that in the absence of nuclear exosome function, oligo(A) tails are lengthened sufficiently by TRAMP to bind oligo-d(T) such that they provide an unbiased view of NNS termination sites.

\section{DNA-binding proteins and nucleosomes mark the sites of NNS termination of snoRNA transcription}

To better understand the determinants that specify the location and width of the NNS termination window, we examined termination behavior at individual snoRNA genes. While the majority of snoRNAs exhibited a broad window of termination, 13 of 52 snoRNA genes exhibited sharp termination peaks (Fig. 2A; central panels). To explore the basis for these peaks, we asked whether any DNA-binding proteins might impact termination and analyzed these loci for the presence of DNase I-resistant sites (DRSs) (Hesselberth et al. 2009). This revealed two distinct classes of termination profiles, depending on the presence or absence of DRSs proximally downstream from xPAT clusters, designated as roadblocked and nonroadblocked, respectively (Fig. 2A). The majority of these roadblocks corresponded to binding sites for the three general regulatory factors (GRFs): Reb1p (SNR8, SNR48, SNR80, SNR81, SNR85, SNR161), Rap1p (SNR39b and SNR72), and Abf1p (SNR45). These initial observations suggested that the GRF class of DNA-binding proteins might be important to promote NNS termination at specific loci.

To test whether snoRNA xPAT peaks upstream of DRSs are indeed generated by Pol II collisions with GRFs, we analyzed nascent elongating transcript sequencing (NET-seq) data, which identifies the RNA 3'-ends associated with Pol II independent of post-transcriptional stability (Churchman and Weissman 2011; Colin et al. 2014). This revealed a build-up of paused Pol II in WT cells proximally upstream of the major xPATs for SNR8 (Reb1p roadblock) and SNR72 (Rap1p roadblock) (Supplemental Fig. S4A). Upon encountering transcriptional stalls, Pol II backtracks and the exposed 3 '-end of the transcript is cleaved by TFIIS (Izban and Luse 1992). Inactivation of TFIIS (yeast Dst1p) thus reveals the locations of the initial pause sites prior to backtracking (Sims et al. 2004). xPAT peaks coincided precisely with NET-seq peaks in $d s t 1 \Delta$ cells, demonstrating that XPATs reveal roadblock-assisted termination occurring in a normal context rather than artifacts of exosome inactivation (Supplemental Fig. S2A). For $S N R 8$, a second prominent peak was detected $27 \mathrm{bp}$ upstream of the major xPAT peak located $12 \mathrm{bp}$ upstream of the Reb1p site. The 27-bp distance between these two xPAT peaks is remarkably consistent with in vitro studies on colliding yeast Pol II elongation complexes, which exhibit a 26- to 27-bp distance between active sites of the leading and trailing Pol II (Saeki and Svejstrup 2009). This suggests that in addition to DNA-binding proteins, a strongly arrested Pol II complex can roadblock a trailing Pol II to promote termination in vivo, and demonstrates that XPATs reveal Pol II dynamics at nucleotide resolution.

Our findings that DNA-bound proteins can promote termination prompted us to examine the relationship between NNS 
A
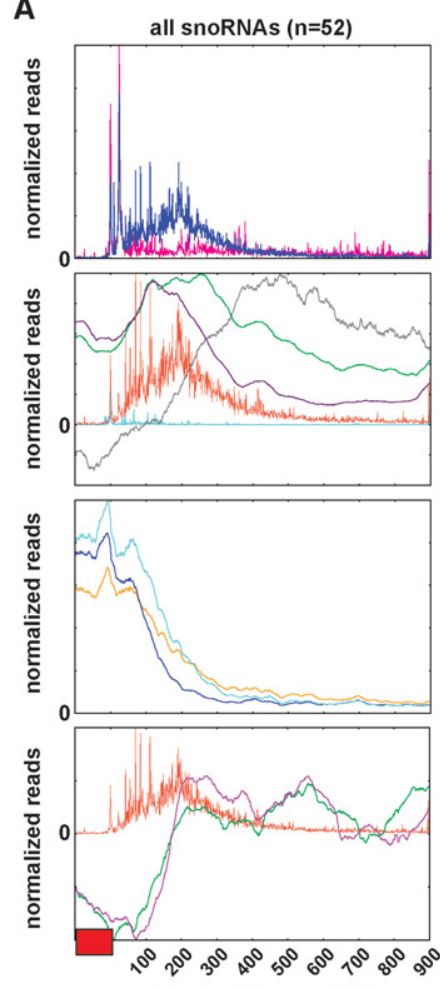

bp downstream snoRNAs
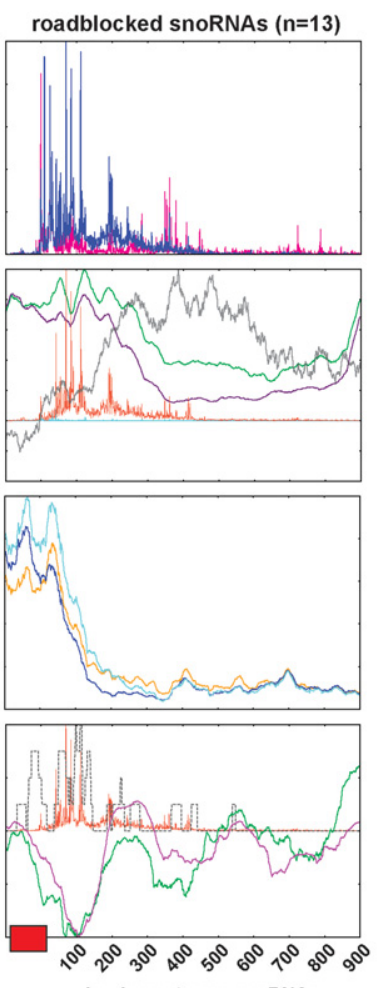

bp downstream snoRNAs non-roadblocked snoRNAs $(\mathrm{n}=39)$
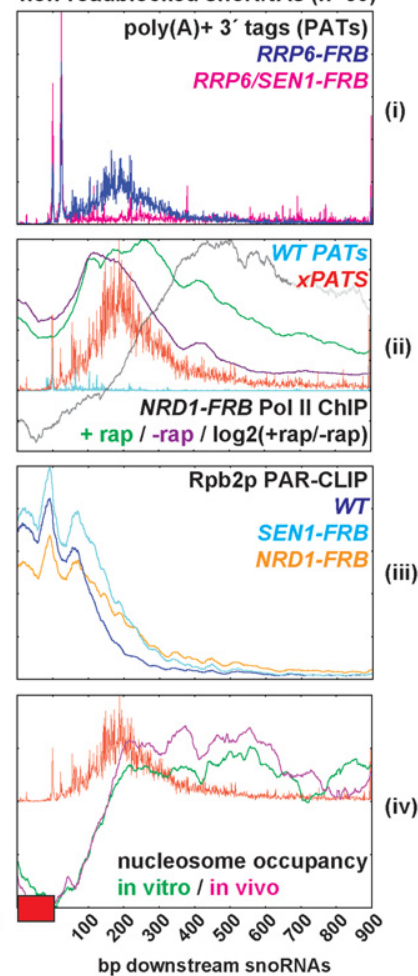

B

non-roadblocked Sen1-dependent XPAT clusters

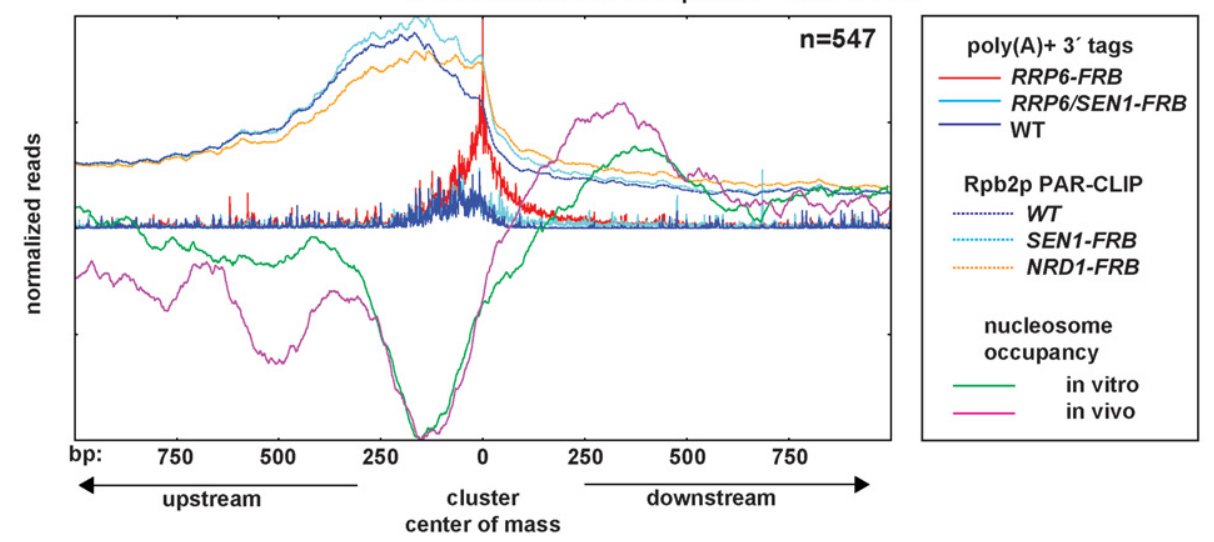

Figure 2. Two distinct classes of NNS-dependent snoRNA transcription termination. (A) Pile-up analysis of a 900-bp region downstream from snoRNA genes for all NNS-terminated snoRNAs (left), snoRNAs with RRP6/DIS3-FRB PAT (xPAT) cluster peaks associated with downstream DRS (roadblocked snoRNAs; middle), and snoRNA XPAT cluster peaks without downstream DRS (nonroadblocked snoRNAs; right). (i,ii,iv) PATs for the indicated strains. (ii) Pol II ChIP signal from NRD1-FRB strains in the absence (purple) or presence (green) of rapamycin, with the gray line indicating the log 2 ratio of rapamycin treated over untreated cells. (iii) Pol II occupancy as assayed by PAR-CLIP of Rpb2p for the indicated strains. (iv) The distribution of xPATs relative to nucleosome occupancy. (iv, middle) DRS signal (dashed gray line) included. The $y$-axes for all plots represent normalized reads. (B) Pile-up analysis on the centers of mass of clusters greater than or equal to twofold up-regulated in RRP6-FRB relative to RRP6/SEN1-FRB and lacking downstream DRS for the indicated data sets. Pol II PAR-CLIP and nucleosome occupancy are depicted as in $A$. The number of clusters included in pile-up analyses is indicated in the upper right $(n=547)$.

termination and nucleosomes, which represent a ubiquitous obstacle for Pol II (Carey et al. 2006; Teves et al. 2014). We analyzed the regions downstream from snoRNAs for nucleosome occupancies detected in vivo or after in vitro assembly (Kaplan et al. 2009). For the 39 nonroadblocked snoRNA genes, the peaks of NNS termination coincided with a sharp increase in nucleosome occupancy (Fig. 2A, iv). Importantly, nucleosome occupancy downstream from NNS peaks is due to intrinsic sequence elements and is not a consequence of Pol II transcription in vivo, as in vitro nucleosome occupancy revealed the same enrichment. Examples of nucleosome-correlated NNS termination sites are shown for the model NNS targets SNR13 and NELO25c, where termination initiates in the first half of a highly occupied nucleosome (Supplemental Fig. S5). Taken together, these observations suggest that chromatin obstacles might generally enhance NNS termination by impeding Pol II progression.

\section{Genome Research}

www.genome.org 
Nucleosomes and DNA-binding proteins specify sites of NNS termination genome-wide

Next we obtained a high-confidence set of nonroadblocked NNS termination sites by screening for xPAT clusters down-regulated greater than or equal to twofold upon nuclear depletion of Sen $1 p$ and lacking downstream DRS (Fig. 2B). To further characterize the behavior of Pol II termination at roadblocked versus nonroadblocked NNS terminators, we separated all xPAT clusters according to the presence or absence of DRSs downstream and compared them to Pol II PAR-CLIP signal obtained after Sen1p or Nrd1p nuclear depletion induced by $30 \mathrm{~min}$ of rapamycin treatment followed by 15 min of crosslinking in the same medium. Aligning nonroadblocked XPAT clusters by their centers of mass revealed a substantial increase in nucleosome signal at the cluster centers, suggesting a global impact of nucleosomes on NNS termination (Fig. 2B). For nonroadblocked xPATs, we observed a sharp drop in Pol II levels at the cluster peaks, consistent with these representing major sites of NNS termination (Figs. 2B, 3A). As a control, we analyzed all nuclear exosome-insensitive PAT cluster peaks, which should primarily correspond to the canonical CPA sites. A gradual increase in Pol II occurred throughout the $250 \mathrm{bp}$ upstream of the PAT peak, consistent with the established pausing of Pol II at the $3^{\prime}$-ends of genes (Glover-Cutter et al. 2008; Nojima et al. 2015). However, no additional increase in Pol II occurred upon depletion of Sen $1 p$, consistent with its dispensability for CPA-mediated termination (Schaughency et al. 2014). A slight increase in Pol II occurred downstream from poly(A) sites upon Nrd1p depletion, but it is unclear if this is due to a direct role of Nrd1p in CPA termination or to an indirect consequence of Nrd1p inactivation (Fig. 3A).

Overall, 367 xPAT clusters were found within 20 bp upstream of a DRS (Supplemental Table S1). Metasite analysis revealed a build-up of Pol II coinciding with xPAT peaks, with a substantial increase upon Nrd1p or Sen1p depletion (Fig. 3A). These data suggest a model in which DNA-binding proteins induce Pol II pausing to promote NNS-mediated release of Pol II. In the absence of NNS, Pol II remains roadblocked at these sites. To demonstrate that increased Pol II upon Nrd1p or Sen1p depletion is specific to NNS termination sites, we analyzed WT PATs upstream of DRS. We observed an increase in Pol II around the cluster peak suggestive of pausing but with no additional effect of Nrd1p or Sen1p inactivation (Fig. 3A).

To uncover determinants directing roadblock-assisted NNSmediated termination, we analyzed the 250 -bp window upstream of the PAT peaks for motifs enriched in XPATs relative to WT PATs. Regardless of the presence of a downstream DRS, motif discovery revealed the extended Nab3p motif UCUUG as the most statistically enriched motif $\left(P=9.7 \times 10^{-10}\right.$ and $1.4 \times 10^{-36}$, with and without a downstream DRS, respectively) (Bailey 2011). This motif has previously been shown to be enriched upstream of natural NNS termination sites and is critical for termination of evolved NNS substrates (Creamer et al. 2011; Porrua et al. 2012; Schaughency et al. 2014). Analysis of the frequency of NNS motifs revealed that UCUUG was more than twofold enriched upstream of xPATs relative to WT PATs, with Nrd1p motifs exhibiting little to no enrichment, consistent with previous observations (Fig. 3B; Schaughency et al. 2014). None of the reverse complements showed enrichment to the levels of the extended Nab3p motif, suggesting that UCUUG is a major determinant for NNS termination genome-wide. 
Roadblocks and the NNS machinery cooperate to promote efficient termination of transcription

Overall, the most abundant xPATs generated by roadblocks were found downstream from snoRNAs (Supplemental Table S1). The decrease of roadblock xPAT signal upon Sen1p inactivation suggested that Reb1p might act in concert with the NNS termination pathway to promote termination (Fig. 2). To dissect the mechanism of roadblock-assisted NNS termination, we analyzed the Reb1p roadblock downstream from the SNR161 gene (Fig. 4A). We hypothesized that efficient Reb1-mediated snoRNA termination might be critical to prevent transcriptional interference of the PDX3 gene, as its transcription start site (TSS) is only $80 \mathrm{bp}$ downstream (Pelechano et al. 2013). To confirm that Reb1p assists in termination of SNR161, we codepleted Reb1p and Rrp6p. This revealed a reduction in the roadblocked XPAT peak and an accumulation of xPATs extending into the ORF of $P D X 3$, indicative of defective termination (Fig. 4A). As an additional control, we mutated the TTACCCG Reb1p motif to TTACAAG, which abolishes Reb1p binding (Colin et al. 2014). This mutation led to the accumulation of a readthrough transcript and to a broad range of 3'-extensions for snR161 in cells lacking Rrp6p (Fig. 4B, mut lanes, probes 1 and 2). Replacing the Reb1p binding site with an RNase III (Rnt1p) cleavage signal (RCS) prevented the accumulation of the readthrough transcript and led to a loss of the NNS termination products, demonstrating that the lengthened transcripts corresponded to 3 '-end extensions of snR161 (Fig. 4B; RCS lanes, probe 2). We also identified xPATs originating from the opposite strand, suggesting that Reb1p can function as a bidirectional roadblock (Fig. 4A). Mutation of the Reb1p binding site led to increased levels of anti-sense transcripts reading through SNR161 (Fig. 4B). As expected, these anti-sense transcripts were unaffected by the strand-specific RCS.

Genome-wide analysis of xPATs upstream of DRSs revealed roadblocks at 77 Reb1p sites, 34 Rap1p sites, and 20 Abf1p sites ( $\geq 2$ rpm) (Supplemental Table S1). Individual examples of Rap1p and Abf1p roadblocks for cryptic Pol II transcripts are shown in Supplemental Figure S6. Meta-analysis of xPATs and NET-seq signal upstream of all GRF binding sites demonstrated significant Pol II pausing, coinciding with xPAT peaks upstream of a DRS (Supplemental Fig. S7). In contrast, bind-

B

D
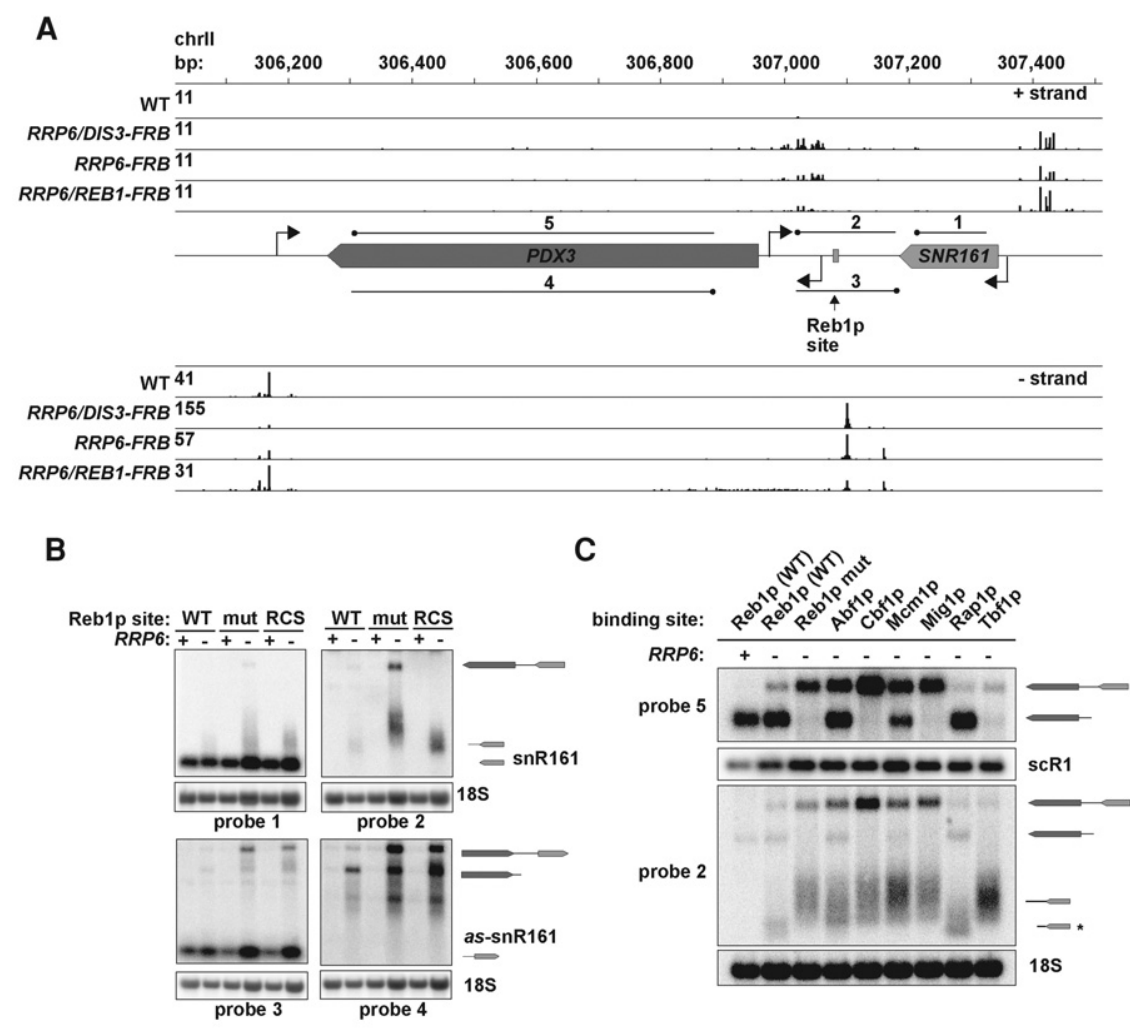

E
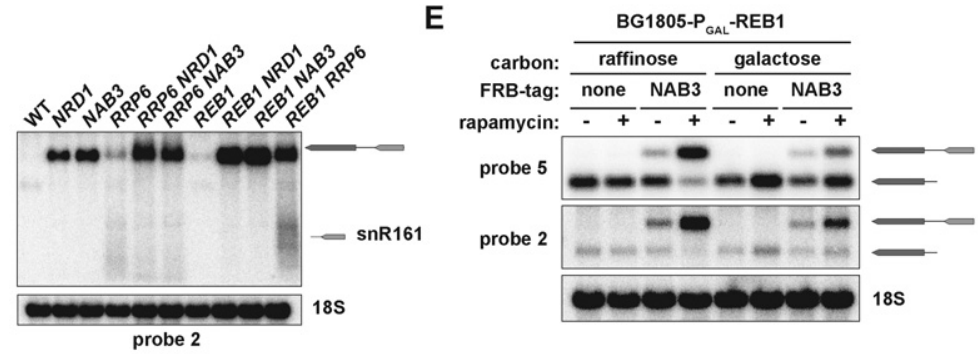

Figure 4. Cooperation between Reb1 $p$ and NNS factors for Pol II transcription termination of SNR161. (A) Genome browser view of the SNR161-PDX3 region. The $y$-axis indicates reads per million (rpm) of PATs for the indicated FRB-tagged strains after $1 \mathrm{~h}$ of rapamycin treatment. (B) Northern blot analysis of sense and anti-sense transcripts at the SNR161 locus. The Reb1 $\mathrm{p}$ binding site in the SNR161 terminator was mutated (mut) or replaced with a cleavage site for the yeast RNase III homolog Rnt1p (RCS). Probes 1 and 2 detect the negative strand transcripts (sense to snR161), while probes 3 and 4 detect positive strand transcripts (anti-sense to snR161). 18S rRNA is shown as a loading control. (C) Northern blot analysis on $r r p 6 \Delta$ with a Reb1 $p$ binding site mutation (TTACCCG $\rightarrow$ TTACAAG) or replacement with indicated proteins and their respective binding sites: Abf1p, TTTGATCGCTTTGTACGTGC; Cbf1p, TTITTATCATGTGACTTATG; Mcm1p, TTACCTAATTAGGTAA; Tbf1p, TTCTTAGGGTTAAATA; Mig1p, AAGCTGAAAATCTGGGGAAG; and Rap1p, AGCAACACCCAGACATTACA. The Tbf1 p binding site inadvertently introduced an additional TCTT motif for Nab3p (underlined). Probe 2 detects 3 '-extended snR161 precursors, while probe 5 detects mature PDX 3 mRNA and the SNR161-PDX3 readthrough product. $(D)$ The indicated anchor away strains were treated with $1 \mu \mathrm{g} / \mathrm{mL}$ rapamycin for $1 \mathrm{~h}$ and Northern blots probed for the region downstream from SNR161 (probe 2). (E) Effect of Reb1p overexpression on PDX3 and SNR161-PDX3 expression upon Nab3 nuclear depletion. WT and NAB3-FRB strains were transformed with the BG1805 plasmid harboring a galactose-inducible REB1. Strains were grown in raffinose to mid-log phase and then were either treated with $1 \mu \mathrm{g} / \mathrm{mL}$ rapamycin for $1 \mathrm{~h}$ (left four lanes) or shifted to galactose for $2 \mathrm{~h}$ prior to the addition of rapamycin (right four lanes). Probe 2 detects the SNR161-PDX3 readthrough transcript with partial overlap with PDX3 $5^{\prime}$ UTR. Probe 5 detects mature PDX3 mRNA and the readthrough product.

ing sites for the subtelomeric anti-silencing factor Tbf1p and the transcription factor $\mathrm{Mcm} 1 \mathrm{p}$ revealed no detectable Pol II pausing and little DRS signal. The centromere-binding factor Cbf1p exhibited a subtle accumulation of Pol II, but not to the extent of the

\section{Genome Research}

www.genome.org 
GRFs. Furthermore, other than TFIIIB (see below), analysis of sequences downstream from roadblocked xPATs did not identify motifs for other DNA-binding proteins (Supplemental Table S1). We conclude that GRFs possess an ability to promote Pol II termination that is unique among the cellular repertoire of DNA-binding proteins.

We next tested a panel of well-characterized binding sites for GRF and non-GRF DNA-binding proteins for their ability to roadblock SNR161 transcription. We replaced the Reb1p site with sites for Rap1p, Abf1p, Cbf1p, Tbf1p, Mig1p, or Mcm1p and then deleted RRP6 and probed for roadblocked and 3'-extended NNS products. Mutation of the Reb1p site led to loss of roadblocked SNR161 and accumulation of heterogeneous 3'-ends reminiscent of nonroadblocked termination (Fig. 4C, probe 2). The Reb1p mutation also led to loss of mature PDX3 mRNA at the expense of a readthrough product, indicating dependence of $P D X 3$ transcription on Reb1p (Fig. 4C, probe 5). The GRF Rap1p was able to fully roadblock SNR161 and restore PDX3 levels. None of the other factors were able to rescue loss of Reb1p, although the NNS termination window generated by the Abf1p site shifted to shorter $3^{\prime}$ extensions than other sites. The Tbf1p binding site prevented readthrough, but closer inspection of the binding site revealed the inadvertent introduction of an additional TCTT Nab3p motif, possibly leading to more efficient NNS termination (Fig. 4C). Whereas Mcm1p only slightly rescued PDX3 levels, Abf1p fully rescued $P D X 3$ despite both yielding similar levels of readthrough. This indicates that Pol II readthrough does not necessarily lead to silencing and suggests that Abf1p, despite being obligatorily dislodged by Pol II during SNR161 readthrough, is still able to maintain a chromatin environment that promotes full PDX3 transcription. It is possible that Abf1p exhibits rapid rebinding or establishes long-lasting effects on the local chromatin that outlive its binding residence time.

To test whether NNS promotes roadblock termination for SNR161, we depleted Nrd1p, Nab3p, and Reb1p from the nucleus for $1 \mathrm{~h}$ in combination with Rrp6p (Fig. 4D). Previous studies had demonstrated a near-complete loss of NNS function and depletion of Nrd1p from the nucleus with $1 \mathrm{~h}$ of rapamycin treatment (Schulz et al. 2013; Castelnuovo et al. 2014). Nrd1p or Nab3p depletion enabled Pol II to elongate through the intact Reb1p site to yield a substantial increase in readthrough transcript. This observation is in agreement with increased levels of Pol II PARCLIP signal downstream from roadblocks upon Nrd1p or Sen1p depletion (Fig. 3). The depletion of Reb1p and Rrp6p led to both readthrough product and the characteristic spread of NNS termination signal. We also analyzed Reb1p roadblock-mediated termination of RPL9B, which undergoes autoregulation in an NNSdependent manner (Supplemental Fig. S8; Gudipati et al. 2012). We found a much greater dependence on termination for Nab3p than Nrd1p, consistent with the presence of three separate TCTTG Nab3p motifs in the 150-bp window upstream of the Reb1p site (Supplemental Fig. S8). In the absence of Reb1p, the majority of transcription terminated in an NNS-dependent manner within 100 bp downstream.

The observation that NNS inactivation results in Pol II reading through the Reb1p site suggests that Pol II is able to evict Reb1p. As Pol II elongation is transient, GRF rebinding should then depend on the concentration of unbound GRF. Consistent with this hypothesis, overexpression of exogenous Reb1p rescued the loss of PDX3 mRNA and diminished levels of readthrough upon Nab3p depletion (Fig. 4E). These data suggest that initial eviction of GRFs by Pol II occurs in a manner that is inhibited by
NNS signals. After GRF eviction, subsequent rounds of GRF-unimpeded transcription ensue until the GRF rebinds in a manner that is dependent on the concentration of free GRFs.

GRFs, nucleosomes, and TFIIIB (addressed below) were previously shown to block the progression of DNA Pol $\delta$ during DNA replication (Smith and Whitehouse 2012).To test how individual GRFs and non-GRFs compare in their ability to roadblock Pol II and Pol $\delta$, we plotted the $3^{\prime}$-ends of Pol $\delta$ fragments that accumulate upon DNA ligase I (CDC9) inactivation alongside Pol II PARCLIP signal, and found the expected accumulation of Pol II and Okazaki fragment $3^{\prime}$-ends proximally upstream of GRF sites and, to a lesser extent, Cbf1p sites (Supplemental Fig. S9). We found no significant enrichment of Okazaki fragment 3 '-ends at Tbf1p or Mcm1p sites. Together, these results demonstrate that all three GRFs roadblock both NNS-targeted Pol II and Pol $\delta$ during lagging strand synthesis.

\section{The NNS pathway dictates the fate of Pol II collisions with GRFs}

Although GRFs can promote NNS termination, many GRF binding sites are positioned within transcribed regions where they do not roadblock Pol II (Wang and Warner 1998; Rhee and Pugh 2011; Colin et al. 2014; Kasinathan et al. 2014). Plotting 1270 verified Reb1p binding sites across binned ORF transcripts (ORF-Ts) revealed the frequent occurrence of Reb1p sites throughout ORFs and in $3^{\prime}$ UTRs (Fig. 5A). Analysis of xPATs accumulating upstream of Reb1p sites revealed 322 sites promoting roadblock termination ( $\geq 1 \mathrm{rpm}$ ) (Fig. 5B; Supplemental Table S3). Roughly half of the Reb1p roadblocks were identified in intergenic regions and CUTs/SUTs/XUTs, consistent with the previously proposed role for Reb1p in restricting cryptic transcription (Colin et al. 2014). Approximately one-third of the roadblocks were found within the transcribed portions of protein coding genes, suggesting widespread regulation of transcription elongation within genes by Reb1p roadblock.

In total, we identified 495 Reb1p sites not leading to Pol II roadblock with evidence of readthrough (see Methods) (Supplemental Table S3). The observation that inactivating NNS abolished roadblock termination and led to high levels of Pol II readthrough at SNR161 and RPL9B raised the possibility that a scarcity of NNS signals may promote readthrough at natural GRF sites. To test this, we analyzed NNS signals upstream of GRF binding sites promoting roadblock relative to those permitting readthrough (Supplemental Table S3). UCUUG was enriched three- to eightfold, with other NNS signals enriched approximately 1.5-fold (Fig. 5C). As a control, we analyzed the reverse complements of NNS motifs and the Hrp1p/Nab4p motif UAUAUA, most of which were slightly depleted. Overall, we did not find a preferred orientation or motif variant for GRF sites exhibiting roadblock versus readthrough behavior, suggesting that NNS signals are the primary determinant for roadblocking Pol II at individual sites (Supplemental Fig. S10; Supplemental Table S3). To further investigate the behavior of Pol II at roadblock and readthrough Reb1p sites, we plotted Pol II PAR-CLIP signal around Reb1p sites exhibiting the TTACCGG consensus motif. Depletion of either Nrd1p or Sen1p led to a substantial increase in Pol II upstream of consensus Reb1p roadblocks and a subtle increase in the $\sim 100 \mathrm{nt}$ downstream (Fig. 5D). As Pol $\delta$ does not synthesize RNA and should be immune to NNS signals, we reasoned that sites with Pol II readthrough should still roadblock DNA Pol $\delta$. Indeed, we found the same prominent peak of Okazaki fragment 3'-ends at both Pol II roadblock and readthrough sites (Fig. 5D). 
A

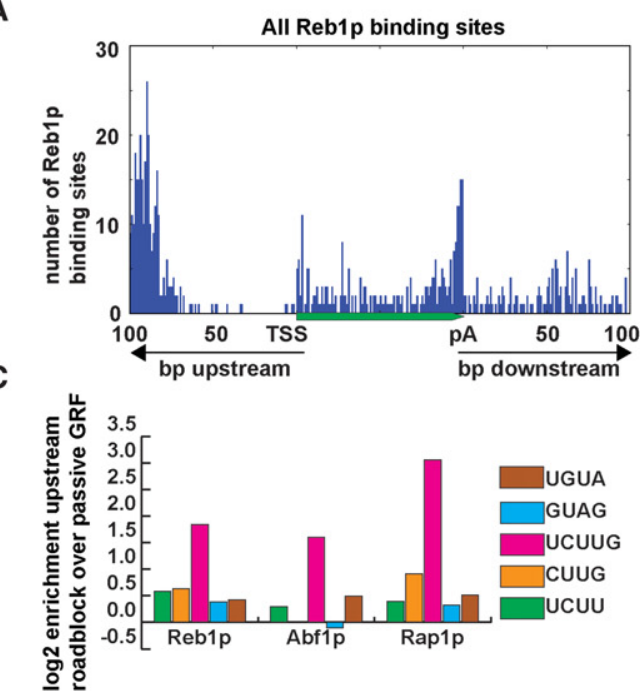

D

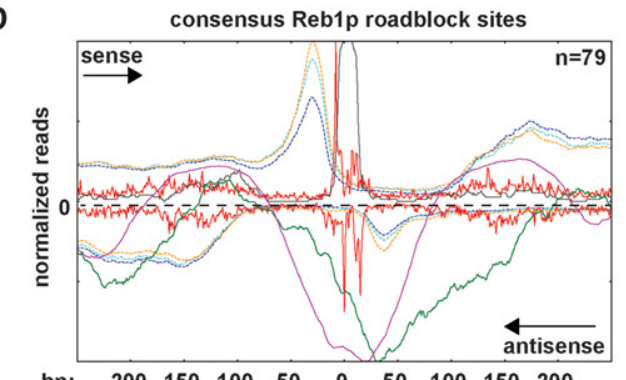

bp: $\begin{array}{lllllllll}200 & 150 & 100 & 50 & 0 & 50 & 100 & 150 & 200\end{array}$
B
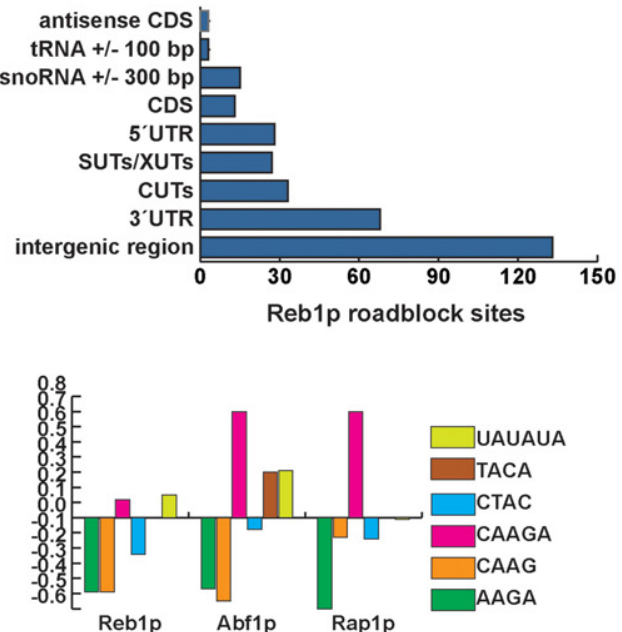

Reb1p

consensus Reb1p readthrough sites

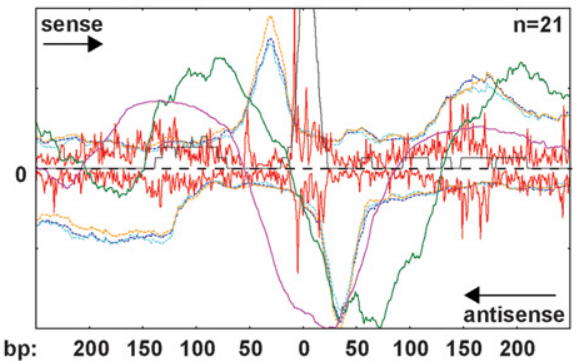

$\mathbf{E}$

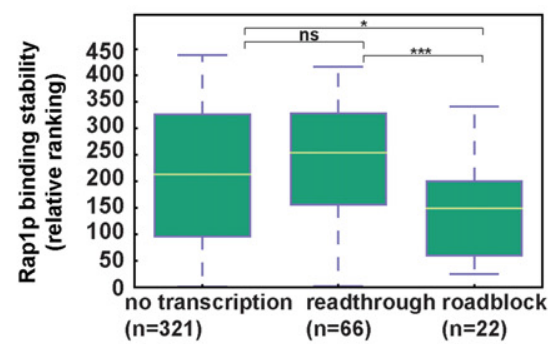

$\mathbf{F}$

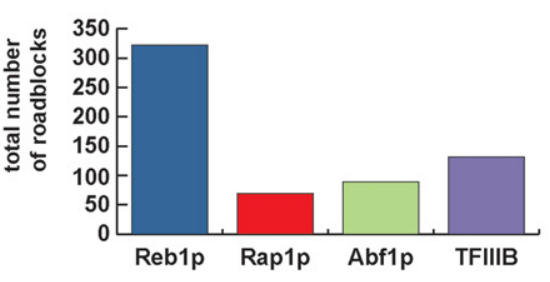

Figure 5. NNS termination signals globally specify the fate of Pol II collisions with general regulatory factors (GRFs). ( $A$ ) Distribution of all Reb1 p binding sites within ORF-Ts (binned into 100 regions) and their flanking 100-bp regions. (B) Genomic distribution of xPAT clusters with peaks proximally upstream of experimentally validated Reb1 $p$ binding sites (see Methods). (C, left) Motif enrichment upstream of binding sites for the GRFs Reb1 p, Abf1p, and Rap1p. One hundred-nucleotide sequences upstream of roadblock sites and readthrough GRF sites were analyzed for Nrd1p (UGUA and GUAG) and Nab3p (CUUG, UCUU, and UCUUG) motifs. The $y$-axis plots the $\log _{2}$ motif enrichment upstream roadblock versus readthrough sites. (Right) Enrichment of the reverse complements of Nrd1 $p$ and Nab3p motifs and the UAUAUA Hrp1p/Nab4p motif as controls. (D) Pol II PAR-CLIP and Pol $\delta$ Okazaki fragment $3^{\prime}$-ends around Reb1 $\mathrm{p}$ sites with the consensus motif (TTACCCGG). The signal above the zero line represents the sense strand with respect to the direction of Pol II roadblock (left) or readthrough (right) for each Reb1 p site, while the signal from the opposite strand is plotted below the zero line. (E) Rap1 p binding sites were grouped according to readthrough behavior, roadblocking behavior, or no convergent Pol II transcription (see Methods). The stability rankings for each Rap1p binding site were obtained from a previous study (Lickwar et al. 2012). Box plots show median stability ranking (green horizontal line) and lower and upper quartile for the three classes of Rap1p binding sites. $\left.{ }^{* * *}\right) P<0.001,\left(^{*}\right) P<0.05$, (ns) not significant. $(F)$ Global distribution of Pol II roadblocks for each DNA-binding factor. Roadblocks are called according to the presence of xPATs within a window 5-25 bp upstream of the binding site ( $\geq 1$ rpm, greater than or equal to twofold up-regulation over WT).

After demonstrating that Pol II can elongate through GRF sites, we predicted that NNS might influence GRF binding dynamics by modulating GRF eviction, particularly at loci with high tran- scriptional activity. A previous study measured Rap1p binding dynamics through competition ChIP and found a wide range of dissociation rates, or mean residence times (Lickwar et al. 2012). 
Importantly, residence time was a better predictor for Rap1p function than occupancy measured by traditional ChIP. To study how Rap1p dynamics might be affected by Pol II collision, we screened Rap1p sites for roadblock and readthrough behavior. Of 439 Rap1p sites, we found 88 sites with evidence for either roadblock or elongation (Supplemental Table S3). Rap1p sites leading to roadblocks exhibited a higher median ranking for Rap1p binding stability and higher levels of NNS signals relative to sites exhibiting readthrough (Fig. 5D,E). Taken together, our results suggest that the NNS pathway can control the binding dynamics of GRFs through influencing the outcome of Pol II collisions.

\section{Pervasive termination of Pol II by the Pol III transcription factor TFIIIB}

Overall, 131 out of 367 roadblocked xPATs were due to the presence of GRFs ( $\geq 2 \mathrm{rpm}$ ) (Supplemental Table S1). Analysis of all GRF sites for xPATs upstream revealed over 400 GRF roadblocks genomewide, with Reb1p being responsible for the majority of these roadblocks $(\geq 1$ rpm) (Fig. 5F). In addition to the GRFs, we noticed that binding sites for the Pol III transcription factor TFIIIB were highly represented downstream from roadblocked xPATs (Fig. 5F; Supplemental Table S1). Pol III transcription was previously suggested to suppress intergenic Pol II transcription, but the precise mechanism of suppression was not determined (Korde et al. 2014; Wang et al. 2014). To confirm whether TFIIIB sites directly roadblock Pol II, we aligned xPATs near all tRNA genes and found a prominent peak centered 65 bp upstream (Fig. 6A).

TFIIIB footprints 10-40 bp upstream of TSSs, which are located $\sim 13$ bp upstream of mature tRNA 5' ends (Joazeiro et al. 1996; Grove et al. 2002). Therefore, the 65-bp peak is consistent with a collision between Pol II and TFIIIB, as the distance between the Pol II active site and the leading edge of Pol II is 12 bp (Saeki and Svejstrup 2009). NET-seq revealed substantial peaks for paused and backtracked Pol II 65 and 72 bp upstream, respectively, with xPAT termination peaks coinciding with the position of stalled rather than backtracked Pol II (Fig. 6A).

To test how Pol III impacts Pol II termination by NNS, we focused on SNR53, which exhibits xPATs downstream from $t G$ (GCC)E (Fig. 6B). To determine the relative contributions of $t G$ (GCC)E and NNS in promoting SNR53 termination, we codepleted either Bdp1p (an essential subunit of TFIIIB) or Sen1p from the nucleus in combination with Rrp6p. Nuclear depletion of either factor led to loss of NNS termination at $t G(G C C) E$ and resulted in the termination at a downstream NNS-independent site (Fig. 6B). Depletion of Nrd1p or Nab3p also resulted in low levels of SNR53 readthrough (Fig. 6C). These results suggested that NNS is dependent on tRNA transcription for efficient SNR53 termination. The results obtained by nuclear depletion of Bdp1p were confirmed by deleting $t G(G C C) E$ in WT and $r r p 6 \Delta$ backgrounds (Fig. 6C).

We next tested whether xPATs upstream of TFIIIB are generally dependent on both NNS and TFIIIB binding. Bdp1p depletion resulted in substantial loss of roadblocked xPATs, with Sen1p depletion conferring only a slight reduction, potentially indicating lesser dependence of TFIIIB roadblocks than GRF roadblocks on NNS (Fig. 7A). Nuclear depletion of either Bdp1p or Sen1p led to the emergence of a new peak coinciding with tRNA TSSs, which are situated 17-19 bp upstream of the box A sequence bound by TFIIIC (Harismendy et al. 2003). This suggests that TFIIIC and TFIIIB are both capable of roadblocking Pol II, with TFIIIB serving as the primary roadblock for Pol II. Overall, we found evidence for TFIIIB roadblocking Pol II at nearly half of all tRNA genes (132/272, $\geq 1 \mathrm{rpm})$, with no tRNA genes showing evidence for Pol II readthrough (Supplemental Table S3). 
A
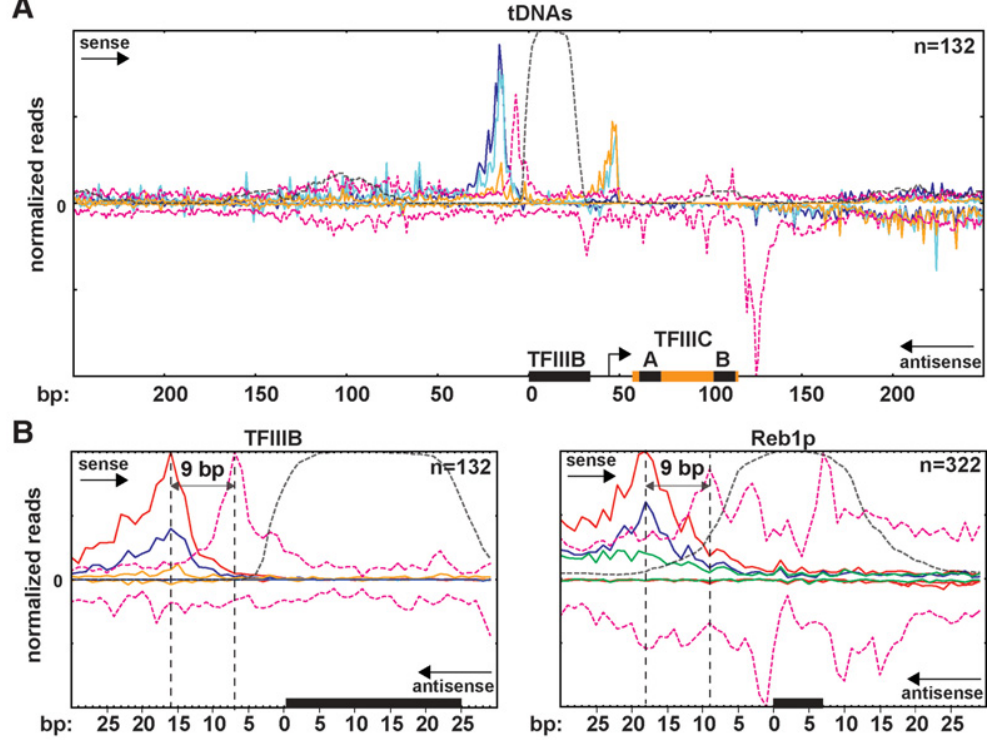

RRP6-FRB RRP6/DIS3-FRB RRP6/SEN1-FRB RRP6/BDP1-FRB RRP6/REB1-FRB

DNase I resistant regions Okazaki fragment $33^{\circ}$-ends

Figure 7. Common DNA-binding factors promote termination of Pol II and lagging strand DNA synthesis. (A) Pile-up analysis of xPAT signal with Okazaki fragment 3 '-ends for all tRNA genes functioning as Pol II roadblocks, centered on the upstream edge of TFIIIB binding sites. The black boxes labeled " $A$ " and " $B$ " correspond to the approximate locations of the box $A$ and box $B$ sequences bound by the transcription factor TFIIIC. Signal above the zero line represents the sense strand with respect to each tRNA gene, while signal below the zero line is anti-sense. (B) Comparison between roadblocked Pol II $3^{\prime}$-ends (XPATs) and roadblocked Pol $\delta$ 3'-ends (Okazaki fragments) for TFIIIB (left) and Reb1p (right) sites. The black bar indicates the TFIIIB footprint (left) and binding motif for Reb1 p (right). (n) Number of roadblocks observed genome-wide for each class. For Reb1 p, signal is oriented relative to the strand of roadblocked transcription.

\section{Common chromatin obstacles terminate both RNA Pol II and DNA Pol $\delta$}

A previous study demonstrated that in addition to nucleosomes and GRFs, tRNA genes also terminate lagging strand synthesis by DNA Pol $\delta$ (Smith and Whitehouse 2012). To compare Pol $\delta$ and Pol II termination at tRNA genes, we plotted xPATs and the $3^{\prime}$ ends of Okazaki fragments around TFIIIB sites (Fig. 7A). This revealed that both Pol II and Pol $\delta$ are roadblocked $16 \mathrm{bp}$ and $7 \mathrm{bp}$, respectively, from the edge of the TFIIIB footprint (Fig. 7A,B). We observed the same 9-bp distances between Pol II RNA and Pol $\delta$ DNA 3 '-ends at Reb1p roadblock sites, suggestive of a characteristic difference in the distance between active site and leading edge of these polymerases during collisions with chromatin obstacles (Fig. 7B). Two major Pol $\delta$ peaks anti-sense to tRNA genes were not detected for Pol II. The first resides on the other side of the TFIIIB binding site and suggests that TFIIIB roadblocks Pol $\delta$ in both directions (Fig. 7A). The second peak occurs at the 3 '-end of tRNA genes and may indicate a collision between DNA Pol $\delta$ and TFIIIC subunits bound at box B. The lack of a significant peak at this location for Pol II may be due to the presence of actively transcribing Pol III at tRNA genes preventing Pol II from approaching TFIIIC, consistent with a recent report that Pol III termination typically occurs over a broad region downstream from tDNA (Turowski et al. 2016). As DNA synthesis by Pol $\delta$ occurs in the context of replication fork movement, it is possible that rapid TFIIIC and TFIIIB assembly on newly synthesized DNA prior to the initiation of Pol III transcription blocks Pol $\delta$.

\section{Discussion}

Chromatin obstacles promote NNS-

The NNS pathway is critical for controlling pervasive Pol II activity and limiting the accumulation of cryptic transcripts. Transcription termination by NNS occurs at stochastic sites over a broad genomic window and is reported to be most efficient during the early phase of transcription when Pol II CTD is enriched for phosphorylated serine 5 (Vasiljeva and Buratowski 2006). However, the mechanisms specifying the precise locations of NNS termination across the genome are not well understood. Previous work demonstrated that a kinetic competition between Sen1p helicase activity and Pol II elongation rate influences the size of the termination window (Hazelbaker et al. 2013). Here we show that endogenous NNS targets differ substantially in the width of the termination window and that diverse types of roadblocks mediate NNS termination. GRFs and TFIIIB promote termination at defined positions with sharp peaks, while nucleosomes exert a global influence on preferred regions of NNS termination (Fig. 2). Our data favor a model in which the NNS pathway and chromatin obstacles cooperate to induce Pol II termination. A Pol II elongation complex preloaded with NNS factors may be inherently less processive and more prone to terminate at roadblocks. On the other hand, pausing induced by roadblocks may favor the recognition of NNS signals in the nascent RNA before elongation proceeds.

Reb1p was previously demonstrated to restrict cryptic transcription by roadblock termination of Pol II in an NNS-independent manner (Colin et al. 2014). The proposed mechanism involved ubiquitylation of Pol II by Rsp5p, analogous to the mechanism used to remove Pol II at sites of DNA damage (Beaudenon et al. 1999). This previous report utilized transcriptional repression of Nrd1p to inactivate NNS, and found no Pol II termination defect at a Reb1p roadblock reporter. Our data suggest that Nab3p plays a more significant role in NNS termination than Nrd1p and that some roadblock targets (e.g., $R P L 9 B$ ) are relatively insensitive to Nrd1p inactivation while sensitive to Nab3p inactivation (Supplemental Fig. S8). Our observation that Reb1p and other GRFs promote termination in an NNS-dependent manner suggests that roadblocked Pol II can be removed independently of ubiquitylation. This is consistent with a recent report that distinct pathways of Pol II removal exist for DNA damage-dependent and DNA damage-independent stalls (Karakasili et al. 2014).

The NNS pathway dictates roadblocking behavior and influences the binding dynamics of GRFs

Through establishing chromatin domains, GRFs coordinate diverse DNA-based activities, including transcription activation or repression and DNA replication and repair (Fourel et al. 2002).

\section{Genome Research}

www.genome.org 
Our results suggest that all three GRFs (Reb1p, Rap1p, and Abf1p) function to block pervasive Pol II transcription. However, GRF binding sites are often located within ORFs and 3' UTRs, raising the question as to how GRFs promote Pol II termination at some sites and allow elongation at others. We demonstrate that the NNS pathway is responsible for this distinction, as inactivating NNS permits elongation through GRF sites that normally roadblock Pol II (Figs. 2, 4). Because nuclear depletion of NNS factors globally impacts gene expression, we cannot strictly exclude the possibility of indirect effects on GRF dynamics at particular loci. However, we find that NNS signals are specifically enriched at GRF sites with roadblock behavior (Fig. 5C). Furthermore, of the subset of Rap1p binding sites with converging Pol II transcription, those exhibiting roadblock behavior harbor more NNS motifs upstream and are more stable than those exhibiting readthrough behavior (Fig. 5C,E). We conclude that an important function of the NNS pathway is to prevent Pol II from displacing GRFs and perturbing their function (Fig. 8).

\section{Common obstacles terminate distinct states of DNA and RNA synthesis}

A previous study demonstrated that lagging strand DNA synthesis occurs as chromatin is reassembled onto Okazaki fragments and that nucleosomes, GRFs, and TFIIIB/tRNA genes prevent excessive strand displacement synthesis by promoting Pol $\delta$ termination (Smith and Whitehouse 2012). These same factors also promote
NNS-mediated Pol II termination. Both leading strand synthesis by Pol $\delta / \varepsilon$ and productive Pol II elongation must traverse nucleosomes and GRF binding sites. An intrinsic sensitivity to roadblocks for Pol $\delta$ in its strand displacement synthesis mode and Pol II in its NNS-targeted mode provides a simple mechanism to limit pervasive nucleic acid synthesis in both contexts. Overall, our results suggest that nucleosomes exert a broad influence on NNS-dependent Pol II termination and that GRFs and TFIIIB function as the most potent roadblocks, although it is possible that other DNAbound factors can roadblock in specific contexts (e.g., Cbf1p). Since NNS motifs are present in virtually all transcripts and are only partially enriched in target versus nontarget RNA, the integration of specific obstacles on the DNA template with varying levels of NNS signals provides a mechanistic explanation for Pol II behavior at different loci (Fig. 8). In summary, this work demonstrates how RNA signals and chromatin obstacles cooperate to terminate Pol II transcription and demonstrates that a common set of roadblocks promotes termination of both DNA and RNA synthesis.

\section{Methods}

All protocols for yeast genetic manipulations, growth conditions, Northern analysis, direct RNA sequencing, and $3^{\prime}$-end poly $(\mathrm{A})^{+}$ RNA-seq library preparation (QuantSeq 3' mRNA-Seq, Lexogen $\mathrm{GmbH}$ ) are included in the Supplemental Methods.

\section{Data processing and metasite analysis}

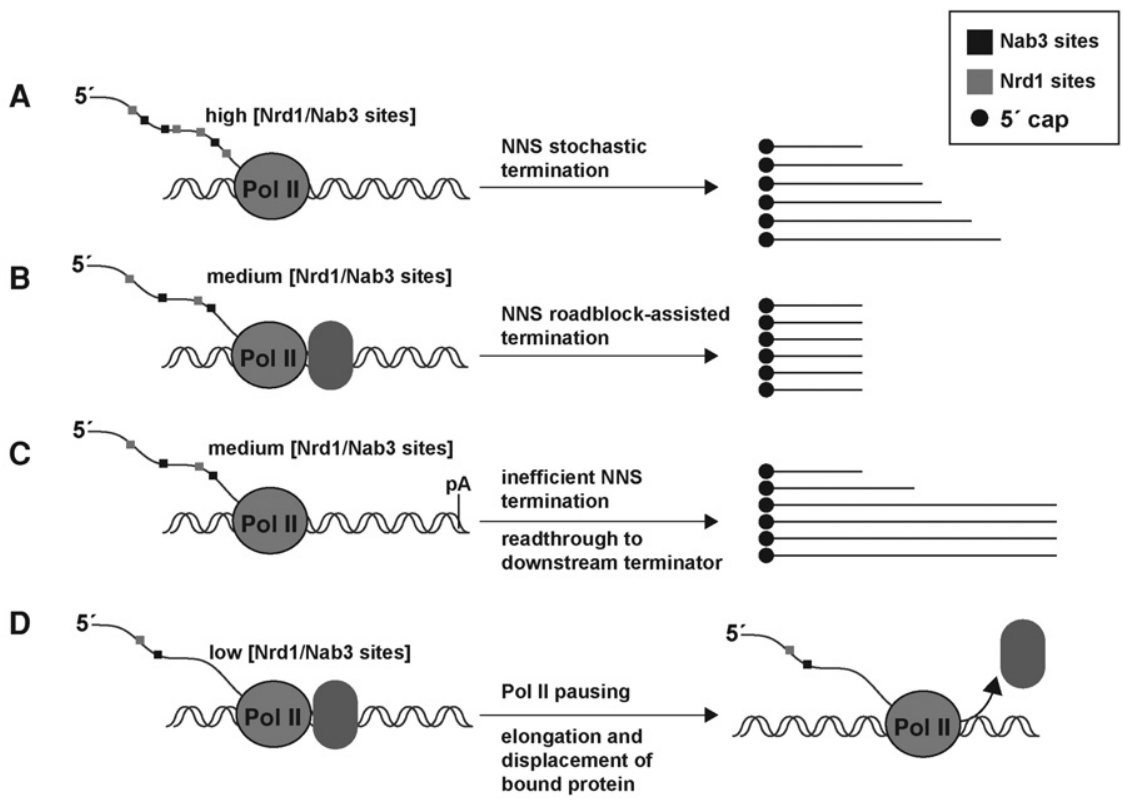

Figure 8. Model for the control of Pol Il elongation and DNA-binding protein dynamics by the NNS pathway. $(A)$ In the presence of a high concentration of Nrd1 p and Nab3p binding sites in the nascent RNA and in the absence of obstacles in the template, Pol II will undergo termination in a stochastic manner over a broad genomic window, resulting in highly heterogeneous $3^{\prime}$-ends with a preference toward nucleosome edges. (B) In the presence of medium (or high) levels of Nrd1 $p$ and Nab3p binding sites and a downstream DNA-binding protein (e.g., GRF, Pol III unit; oval), Pol II will undergo termination at a defined position dictated by the collision of Pol II with the DNA-binding protein and produce a homogenous set of RNA $3^{\prime}$-ends. $(C)$ In the presence of medium levels of Nrd1p and Nab3p binding sites and the lack of any downstream DNA-binding protein, Pol II will undergo inefficient NNS termination and continue reading through until encountering a downstream terminator. In this example, a downstream CPA site is utilized. $(D)$ In the presence of low levels of Nrd1 $p$ and Nab3p binding sites and a downstream DNA-binding factor, Pol II will elongate through the binding site, displacing the DNA-bound protein (oval) from the template.
Clusters were seeded by selecting poly(A) tags (PATs) with $\geq 2 \mathrm{rpm}$ and greater than or equal to fivefold up-regulation after nuclear exosome inactivation (xPATs). PATs were clustered together if within $20 \mathrm{bp}$ of each other and as long as the cluster maintained greater than or equal to twofold up-regulation. The coordinates for cluster peaks were used to define the annotated mapping location for the entire cluster. Detailed descriptions of cluster annotation and identification of motif sites from previous data sets are in the Supplemental Methods. Metasite analysis was conducted by first extracting the signal across genomic windows from a given data set centered around the indicated features, normalizing the sum of the reads in the window to an arbitrary constant, and then aggregating those normalized reads for all the genomic windows. For tracks with only one data set, regions were required to exhibit $\geq 1 \mathrm{rpm}$ for each window prior to normalization. For tracks with multiple data sets (e.g., WT and mutants), the sum of signals for a given region across all data sets was first used to normalize each region prior to signal aggregation, therefore allowing the relative signal between strains to be assessed in the pile-up. For plots showing sense and anti-sense strands, both strands were normalized together for each individual window (Figs. 5D, 7A,B; Supplemental Figs. S7, S9). For binding sites of the indicated 
proteins, the left-to-right direction was oriented with respect to the strand exhibiting the higher XPAT signal. For Figure 5A, the regions from TSSs to poly(A) sites ( $\mathrm{pA}$; as defined from TIF-seq [Pelechano et al. 2013]) were normalized into 100 bins and analyzed together with the upstream and downstream $100 \mathrm{bp}$ for the presence of experimentally validated Reb1p binding sites. All scripts were written in Python 3.5, and plots were generated using the matplotlib module of Python 3.5. The sequence logos in Supplemental Figure S10 were generated with WebLogo version 2.8.2 (Crooks et al. 2004).

\section{Data access}

All raw and processed data from this study have been submitted to the NCBI Gene Expression Omnibus (GEO; http://www.ncbi.nlm. nih.gov/geo/) under accession number GSE75587.

\section{Acknowledgments}

We thank G.-F. Richard for helpful discussions. This study was funded by grant GM061518 from the National Institute of General Medical Sciences to G.F.C.; K.R. was supported by a Philip Whitcome Graduate Fellowship and the National Institutes of Health training program T32GM007185. J.G. was supported by National Institutes of Health training program T32GM007185.

Author contributions: K.R. and G.F.C. conceived the study. K.R., J.G., A.G., and D.N. performed experiments. K.R. performed computational analyses. K.R. and G.F.C. wrote the manuscript.

\section{References}

Allmang C, Kufel J, Chanfreau G, Mitchell P, Petfalski E, Tollervey D. 1999. Functions of the exosome in rRNA, snoRNA and snRNA synthesis. EMBO J 18: 5399-5410.

Bailey TL. 2011. DREME: motif discovery in transcription factor ChIP-seq data. Bioinformatics 27: 1653-1659.

Beaudenon SL, Huacani MR, Wang G, McDonnell DP, Huibregtse JM. 1999. Rsp5 ubiquitin-protein ligase mediates DNA damage-induced degradation of the large subunit of RNA polymerase II in Saccharomyces cerevisiae. Mol Cell Biol 19: 6972-6979.

Briggs MW, Burkard KT, Butler JS. 1998. Rrp6p, the yeast homologue of the human PM-Scl 100-kDa autoantigen, is essential for efficient 5.8 S rRNA 3' end formation. J Biol Chem 273: 13255-13263.

Carey M, Li B, Workman JL. 2006. RSC exploits histone acetylation to abrogate the nucleosomal block to RNA polymerase II elongation. $\mathrm{Mol} \mathrm{Cell}$ 24: $481-487$.

Carroll KL, Pradhan DA, Granek JA, Clarke ND, Corden JL. 2004. Identification of cis elements directing termination of yeast nonpolyadenylated snoRNA transcripts. Mol Cell Biol 24: 6241-6252.

Carroll KL, Ghirlando R, Ames JM, Corden JL. 2007. Interaction of yeast RNA-binding proteins Nrd1 and Nab3 with RNA polymerase II terminator elements. RNA 13: 361-373.

Castelnuovo M, Zaugg JB, Guffanti E, Maffioletti A, Camblong J, Xu Z, Clauder-Münster S, Steinmetz LM, Luscombe NM, Stutz F. 2014. Role of histone modifications and early termination in pervasive transcription and antisense-mediated gene silencing in yeast. Nucleic Acids Res 42: $4348-4362$.

Churchman LS, Weissman JS. 2011. Nascent transcript sequencing visualizes transcription at nucleotide resolution. Nature 469: 368-373.

Colin J, Candelli T, Porrua O, Boulay J, Zhu C, Lacroute F, Steinmetz LM, Libri D. 2014. Roadblock termination by Reb1p restricts cryptic and readthrough transcription. Mol Cell 56: 667-680.

Creamer TJ, Darby MM, Jamonnak N, Schaughency P, Hao H, Wheelan SJ, Corden JL. 2011. Transcriptome-wide binding sites for components of the Saccharomyces cerevisiae non-poly(A) termination pathway: Nrd1, Nab3, and Sen1. PLoS Genet 7: e1002329.

Crooks GE, Hon G, Chandonia J-M, Brenner SE. 2004. WebLogo: a sequence logo generator. Genome Res 14: 1188-1190.

Egecioglu DE, Henras AK, Chanfreau GF. 2006. Contributions of Trf4p- and Trf5p-dependent polyadenylation to the processing and degradative functions of the yeast nuclear exosome. RNA 12: 26-32.

Fourel G, Miyake T, Defossez P-A, Li R, Gilson E. 2002. General regulatory factors (GRFs) as genome partitioners. J Biol Chem 277: 41736-41743.
Fox MJ, Gao H, Smith-Kinnaman WR, Liu Y, Mosley AL. 2015. The exosome component Rrp6 is required for RNA polymerase II termination at specific targets of the Nrd1-Nab3 pathway. PLoS Genet 11: e1004999.

Ghazal G, Gagnon J, Jacques P-É, Landry J-R, Robert F, Elela SA. 2009. Yeast RNase III triggers polyadenylation-independent transcription termination. Mol Cell 36: 99-109.

Glover-Cutter K, Kim S, Espinosa J, Bentley DL. 2008. RNA polymerase II pauses and associates with pre-mRNA processing factors at both ends of genes. Nat Struct Mol Biol 15: 71-78.

Grove A, Adessa MS, Geiduschek EP, Kassavetis GA. 2002. Marking the start site of RNA polymerase III transcription: the role of constraint, compaction and continuity of the transcribed DNA strand. EMBO J 21: 704-714.

Grzechnik P, Kufel J. 2008. Polyadenylation linked to transcription termination directs the processing of snoRNA precursors in yeast. Mol Cell 32: 247-258.

Grzechnik P, Tan-Wong SM, Proudfoot NJ. 2014. Terminate and make a loop: regulation of transcriptional directionality. Trends Biochem Sci 39: $319-327$

Gudipati RK, Neil H, Feuerbach F, Malabat C, Jacquier A. 2012. The yeast $R P L 9 B$ gene is regulated by modulation between two modes of transcription termination. $E M B O$ J 31: 2427-2437.

Harismendy O, Gendrel C-G, Soularue P, Gidrol X, Sentenac A, Werner M, Lefebvre O. 2003. Genome-wide location of yeast RNA polymerase III transcription machinery. EMBO J 22: 4738-4747.

Haruki H, Nishikawa J, Laemmli UK. 2008. The anchor-away technique: rapid, conditional establishment of yeast mutant phenotypes. Mol Cell 31: 925-932.

Hazelbaker DZ, Marquardt S, Wlotzka W, Buratowski S. 2013. Kinetic competition between RNA Polymerase II and Sen1-dependent transcription termination. Mol Cell 49: 55-66.

Hesselberth JR, Chen X, Zhang Z, Sabo PJ, Sandstrom R, Reynolds AP, Thurman RE, Neph S, Kuehn MS, Noble WS, et al. 2009. Global mapping of protein-DNA interactions in vivo by digital genomic footprinting. Nat Methods 6: 283-289.

Izban MG, Luse DS. 1992. The RNA polymerase II ternary complex cleaves the nascent transcript in a $3^{\prime} \rightarrow 5^{\prime}$ direction in the presence of elongation factor SII. Genes Dev 6: 1342-1356.

Joazeiro CA, Kassavetis GA, Geiduschek EP. 1996. Alternative outcomes in assembly of promoter complexes: the roles of TBP and a flexible linker in placing TFIIIB on tRNA genes. Genes Dev 10: 725-739.

Kaplan N, Moore IK, Fondufe-Mittendorf Y, Gossett AJ, Tillo D, Field Y, LeProust EM, Hughes TR, Lieb JD, Widom J, et al. 2009. The DNA-encoded nucleosome organization of a eukaryotic genome. Nature 458: 362-366.

Karakasili E, Burkert-Kautzsch C, Kieser A, Sträßer K. 2014. Degradation of DNA damage-independently stalled RNA polymerase II is independent of the E3 ligase Elc1. Nucleic Acids Res 42: 10503-10515.

Kasinathan S, Orsi GA, Zentner GE, Ahmad K, Henikoff S. 2014. High-resolution mapping of transcription factor binding sites on native chromatin. Nat Methods 11: 203-209.

Korde A, Rosselot JM, Donze D. 2014. Intergenic transcriptional interference is blocked by RNA polymerase III transcription factor TFIIIB in Saccharomyces cerevisiae. Genetics 196: 427-438.

LaCava J, Houseley J, Saveanu C, Petfalski E, Thompson E, Jacquier A, Tollervey D. 2005. RNA degradation by the exosome is promoted by a nuclear polyadenylation complex. Cell 121: 713-724.

Lickwar CR, Mueller F, Hanlon SE, McNally JG, Lieb JD. 2012. Genome-wide protein-DNA binding dynamics suggest a molecular clutch for transcription factor function. Nature 484: 251-255.

Makino DL, Baumgärtner M, Conti E. 2013. Crystal structure of an RNAbound 11-subunit eukaryotic exosome complex. Nature 495: 70-75.

Mitchell P, Petfalski E, Shevchenko A, Mann M, Tollervey D. 1997. The exosome: a conserved eukaryotic RNA processing complex containing multiple $3^{\prime} \rightarrow 5^{\prime}$ exoribonucleases. Cell 91: 457-466.

Neil H, Malabat C, d'Aubenton-Carafa Y, Xu Z, Steinmetz LM, Jacquier A. 2009. Widespread bidirectional promoters are the major source of cryptic transcripts in yeast. Nature 457: 1038-1042.

Nojima T, Gomes T, Grosso ARF, Kimura H, Dye MJ, Dhir S, Carmo-Fonseca M, Proudfoot NJ. 2015. Mammalian NET-seq reveals genome-wide nascent transcription coupled to RNA processing. Cell 161: 526-540.

Ozsolak F. 2014. Quantitative polyadenylation site mapping with singlemolecule direct RNA sequencing. Methods Mol Biol 1125: 145-155.

Ozsolak F, Platt AR, Jones DR, Reifenberger JG, Sass LE, McInerney P, Thompson JF, Bowers J, Jarosz M, Milos PM. 2009. Direct RNA sequencing. Nature 461: 814-818.

Pelechano V, Wei W, Steinmetz LM. 2013. Extensive transcriptional heterogeneity revealed by isoform profiling. Nature 497: 127-131.

Porrua O, Libri D. 2015. Transcription termination and the control of the transcriptome: why, where and how to stop. Nat Rev Mol Cell Biol 16: 190-202.

\section{Genome Research}

www.genome.org 
Porrua O, Hobor F, Boulay J, Kubicek K, D’Aubenton-Carafa Y, Gudipati RK, Stefl R, Libri D. 2012. In vivo SELEX reveals novel sequence and structural determinants of Nrd1-Nab3-Sen1-dependent transcription termination. EMBO J 31: 3935-3948.

Proudfoot NJ. 2011. Ending the message: poly(A) signals then and now. Genes Dev 25: 1770-1782.

Rhee HS, Pugh BF. 2011. Comprehensive genome-wide protein-DNA interactions detected at single-nucleotide resolution. Cell 147: 1408-1419.

Richard P, Manley JL. 2009. Transcription termination by nuclear RNA polymerases. Genes Dev 23: 1247-1269.

Rondón AG, Mischo HE, Kawauchi J, Proudfoot NJ. 2009. Fail-safe transcriptional termination for protein-coding genes in S. cerevisiae. Mol Cell 36: 88-98.

Saeki H, Svejstrup JQ. 2009. Stability, flexibility, and dynamic interactions of colliding RNA polymerase II elongation complexes. Mol Cell 35: 191-205.

Schaughency P, Merran J, Corden JL. 2014. Genome-wide mapping of yeast RNA polymerase II termination. PLoS Genet 10: e1004632.

Schneider C, Kudla G, Wlotzka W, Tuck A, Tollervey D. 2012. Transcriptome-wide analysis of exosome targets. Mol Cell 48: 422-433.

Schulz D, Schwalb B, Kiesel A, Baejen C, Torkler P, Gagneur J, Soeding J, Cramer P. 2013. Transcriptome surveillance by selective termination of noncoding RNA synthesis. Cell 155: 1075-1087.

Sims RJ, Belotserkovskaya R, Reinberg D. 2004. Elongation by RNA polymerase II: the short and long of it. Genes Dev 18: 2437-2468.

Skourti-Stathaki K, Proudfoot NJ, Gromak N. 2011. Human senataxin resolves RNA/DNA hybrids formed at transcriptional pause sites to promote Xrn2-dependent termination. Mol Cell 42: 794-805.

Smith DJ, Whitehouse I. 2012. Intrinsic coupling of lagging-strand synthesis to chromatin assembly. Nature 483: 434-438.

Steinmetz EJ, Conrad NK, Brow DA, Corden JL. 2001. RNA-binding protein Nrd1 directs poly(A)-independent 3'-end formation of RNA polymerase II transcripts. Nature 413: 327-331.

Teves SS, Weber CM, Henikoff S. 2014. Transcribing through the nucleosome. Trends Biochem Sci 39: 577-586.

Tudek A, Porrua O, Kabzinski T, Lidschreiber M, Kubicek K, Fortova A, Lacroute F, Vanacova S, Cramer P, Stefl R, et al. 2014. Molecular basis for coordinating transcription termination with noncoding RNA degradation. Mol Cell 55: 467-481.
Turowski TW, Leśniewska E, Delan-Forino C, Sayou C, Boguta M, Tollervey D. 2016. Global analysis of transcriptionally engaged yeast RNA polymerase III reveals extended tRNA transcripts. Genome Res 26: 933-944.

van Hoof A, Lennertz P, Parker R. 2000. Yeast exosome mutants accumulate 3'-extended polyadenylated forms of U4 small nuclear RNA and small nucleolar RNAs. Mol Cell Biol 20: 441-452.

Vanácová S, Wolf J, Martin G, Blank D, Dettwiler S, Friedlein A, Langen $\mathrm{H}_{\text {, }}$ Keith G, Keller W. 2005. A new yeast poly(A) polymerase complex involved in RNA quality control. PLoS Biol 3: e189.

Vasiljeva L, Buratowski S. 2006. Nrd1 interacts with the nuclear exosome for 3 ' processing of RNA polymerase II transcripts. Mol Cell 21: 239-248.

Venters BJ, Pugh BF. 2009. How eukaryotic genes are transcribed. Crit Rev Biochem Mol Biol 44: 117-141.

Wang KL, Warner JR. 1998. Positive and negative autoregulation of REB1 transcription in Saccharomyces cerevisiae. Mol Cell Biol 18: 4368-4376.

Wang Q, Nowak CM, Korde A, Oh D-H, Dassanayake M, Donze D. 2014. Compromised RNA polymerase III complex assembly leads to local alterations of intergenic RNA polymerase II transcription in Saccharomyces cerevisiae. BMC Biol 12: 89.

Webb S, Hector RD, Kudla G, Granneman S. 2014. PAR-CLIP data indicate that Nrd1-Nab3-dependent transcription termination regulates expression of hundreds of protein coding genes in yeast. Genome Biol 15: R8.

Wilkening S, Pelechano V, Järvelin AI, Tekkedil MM, Anders S, Benes V, Steinmetz LM. 2013. An efficient method for genome-wide polyadenylation site mapping and RNA quantification. Nucleic Acids Res 41: e65.

Wyers F, Rougemaille M, Badis G, Rousselle J-C, Dufour M-E, Boulay J, Régnault B, Devaux F, Namane A, Séraphin B, et al. 2005. Cryptic pol II transcripts are degraded by a nuclear quality control pathway involving a new poly(A) polymerase. Cell 121: 725-737.

Xu Z, Wei W, Gagneur J, Perocchi F, Clauder-Münster S, Camblong J, Guffanti E, Stutz F, Huber W, Steinmetz LM. 2009. Bidirectional promoters generate pervasive transcription in yeast. Nature 457: 1033-1037.

Zhang H, Rigo F, Martinson HG. 2015. Poly(A) signal-dependent transcription termination occurs through a conformational change mechanism that does not require cleavage at the poly(A) site. Mol Cell 59: 437-448.

Received January 23, 2016; accepted in revised form August 18, 2016. 


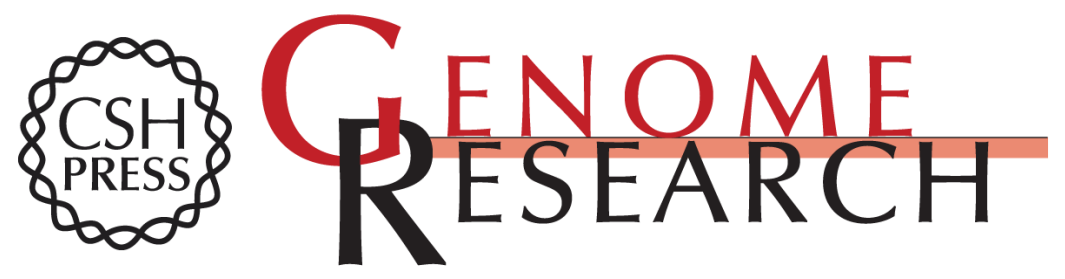

\section{Common genomic elements promote transcriptional and DNA replication roadblocks}

Kevin Roy, Jason Gabunilas, Abigail Gillespie, et al.

Genome Res. 2016 26: 1363-1375 originally published online August 18, 2016

Access the most recent version at doi:10.1101/gr.204776.116

Supplemental Material

References

Creative

Commons

License

Email Alerting Service
http://genome.cshlp.org/content/suppl/2016/09/14/gr.204776.116.DC1

This article cites 68 articles, 21 of which can be accessed free at: http://genome.cshlp.org/content/26/10/1363.full.html\#ref-list-1

This article is distributed exclusively by Cold Spring Harbor Laboratory Press for the first six months after the full-issue publication date (see

http://genome.cshlp.org/site/misc/terms.xhtml). After six months, it is available under a Creative Commons License (Attribution-NonCommercial 4.0 International), as described at http://creativecommons.org/licenses/by-nc/4.0/.

Receive free email alerts when new articles cite this article - sign up in the box at the top right corner of the article or click here.

\section{Affordable, Accurate Sequencing.}

To subscribe to Genome Research go to:

https://genome.cshlp.org/subscriptions 